\title{
Multi-Effect Factor Analysis of Residential Engineering Quality Problems
}

\author{
Xue-liang HOU ${ }^{a}$, Yi WANG \\ Institute of engineering management, North China electric power university, Beijing, China \\ ahou-xl@163.com, bwangyikelven@163.com
}

\begin{abstract}
Keywords: Residence Quality, Evidence Management; Multi-effect Factor.
Abstract. There are many quality problems appearing in residential engineering in recent years. Such residential engineering quality problems are investigated. Through statistical analysis of survey samples, 24 kinds of major quality problems and their corresponding features which often appear in residential engineering in recent years have been found, and the factors result in these residential quality problems can be classified into 11 categories by Delphi method. Using the adjacent matrix method based on reduction to unity, the multi-effect relationship among 11 kinds of factors is analyzed, and the impact degree of every factor to residential engineering problems has been obtained. The research achievement provides the scientific evidence for further solving residential engineering quality problems.
\end{abstract}

\section{Introduction}

With the rapid development of Chinese economy in recent years, people's residential condition and life environment has been changed and improved in big extend. Because the residential qualities related to people's daily life, safety and value of property, people pay more attention to house quality. However, it can be known from some mediums and related research reports that the residential quality situations in china are not so good ${ }^{[1]}$. Some residential engineering accidents appear in some cities and house consumers' complaints also increase rapidly. Such problem not only brings much adverse effect on social stability, but also produces a large amount of economic loss to nation. Thus, it is necessary to analyze the problem in time for seeking an effective solving method.

\section{The Main Problems of Residential Engineering and Their New Feature}

In order to find and understand the existing quality problems in residential construction in recent years, the research group investigated the quality status of residential engineering in China that constructed between 2014 and 2016 with the support of Chinese ministry of construction, the national administration of quality supervision, China consumers association as well as 19 large-scale construction enterprises, and obtained 2113 effective survey samples and relative historical data in 2005 and 2006. Based on statistics results of survey samples, 24 kinds of major quality problems which often appear in residential engineering in recent years have been found. The descriptions and their cumulative reflection frequency of the 24 kinds of quality problems are shown in table 1 .

It can be known from above table 1 and survey results that the whole level of Chinese residential construction quality has been improved obviously through years of efforts, especially, the quality problems in engineering main structure have a decrease in large. Meanwhile, some new following features of residential engineering quality problems have been found through comparative analysis on the cumulative reflection frequency of 24 kinds of quality problems with their corresponding historical data in 2005 and 2006.

(1) The difference of residential quality levels in various regions is very large.

Knowing from the statistics of survey data, although the total residential engineering quality in China has a large improvement in recent years ${ }^{[2]}$, there is a big difference in residential quality levels in various regions. Overall, the engineering quality in economic developed areas is better than in economic underdeveloped areas, the engineering quality in big cities is better than in small and 
Table.1 Statistics of quality problems in residential engineering

\begin{tabular}{|c|l|c|c|c|c|c|}
\hline \multirow{2}{*}{ Order Problems description } & \multicolumn{4}{|c|}{ Cumulative reflection frequency } \\
\cline { 3 - 6 } & & 2014 & 2015 & 2016 & 2005 & 2006 \\
\hline 1 & Cracks of wall, floor, and roof. & 7114 & 5327 & 4271 & 9203 & 8994 \\
\hline 2 & Water percolation in roof, wall, bathroom or kitchen floor. & 6920 & 5306 & 3319 & 7342 & 10003 \\
\hline 3 & Balcony railing disengages or cracks of balcony board. & 2085 & 1477 & 752 & 8133 & 8542 \\
\hline 4 & Water, heating and gas pipe qualities are substandard. & 1173 & 912 & 966 & 4001 & 5347 \\
\hline 5 & Blockage of water, heat and gas pipelines or other facilities. & 2493 & 1702 & 1107 & 8544 & 11432 \\
\hline 6 & Exterior or interior wall's plaster layers peel. & 1817 & 803 & 112 & 3974 & 5125 \\
\hline 7 & Facility like television, telephone, Internet, intercom is failure. & 2951 & 3124 & 4411 & 1114 & 312 \\
\hline 8 & Electrical circuit is unreasonable or leakage. & 1512 & 1181 & 758 & 4399 & 5102 \\
\hline 9 & Damage or deforming of doors or windows. & 3342 & 3018 & 2293 & 5731 & 6112 \\
\hline 10 & Switches of door and window are damaged. & 1556 & 1417 & 2031 & 3231 & 3015 \\
\hline 11 & Substandard soundproof capability of door, window, wall or floor. & 1945 & 2308 & 4530 & 902 & 414 \\
\hline 12 & Substandard insulation heat effect of wall, door and window. & 2828 & 1861 & 2312 & 521 & 993 \\
\hline 13 & Indoor air is polluted by ornament materials. & 1968 & 5323 & 7495 & 246 & 214 \\
\hline 14 & Substandard floor slope in balcony, kitchen or bathroom. & 1453 & 1042 & 751 & 1018 & 2101 \\
\hline 15 & Ceramic tiles of floor, wall, bathroom or kitchen fall off. & 744 & 1333 & 3201 & 594 & 203 \\
\hline 16 & Damp or mildew on walls or floors. & 201 & 155 & 167 & 302 & 451 \\
\hline 17 & Water percolation in basement floor or exterior wall. & 552 & 304 & 151 & 428 & 593 \\
\hline 18 & Flue, rubbish well or vent is blocked up. & 610 & 442 & 201 & 553 & 431 \\
\hline 19 & Deflection of floor is substandard. & 30 & 32 & 67 & 87 & 51 \\
\hline 20 & Indoor equipment and facilities are not securely installed. & 52 & 62 & 40 & 12 & 21 \\
\hline 21 & Inferior materials & 2808 & 4903 & 5721 & 1611 & 1114 \\
\hline 22 & Bathroom stinks. & 302 & 565 & 445 & 371 & 103 \\
\hline 23 & Elevator is unable using properly. & 432 & 499 & 712 & 57 & 8 \\
\hline 24 & Rainwater pipes, sewer pipes or public facilities are damaged. & 1217 & 1633 & 2174 & 23 & 82 \\
\hline
\end{tabular}

medium-sized cities, and the engineering quality in small \& medium-sized cities is better than in towns and villages. Despite there are some high quality residential engineering, the rate of high quality residential engineering in all residential engineering is very lower.

(2) Some new quality problems emerge.

With the development of new materials and new building technology, some quality problems in the past residential engineering have been cured. But some new quality problems emerge with the application of new materials, new technologies and new implement processes, such as failure in communication lines, substandard soundproof capability of door, window, wall and floors, substandard insulation heat effect of wall, door and window and so on. Especially, because of using a large number of new ornament materials in housing building, indoor air pollution have become the most important, significant and very common quality problem, which already becomes a hot complaint point by people.

(3) The residential quality complaint range is enlarging.

Before 2006, consumers rarely make a complaint on the residential public facilities and environment, such complaints in many cities is even zero. But it can be seen from the statistics of survey data that these problems complaints number on the residential public facilities and environment has an obvious increasing in recent years. In some of economic developed cities like Beijing, Shanghai, Shenzhen, this kind of complaint is more than other cities relatively. This shows that consumers not only pay attention on residential quality at present, but also more concerned about the overall quality and living environment.

\section{Group Factor Analyses of Residential Quality Problems}

In order to seek and find the reasons of these residential quality problems, and put forward the 
scientific evidences for further solving the problems, above 24 kinds of quality problems are discussed by Delphi method after inviting 12 engineering experts. These experts think through 13 round of discussion that the factors result in these residential quality problems can be classified into 11 categories, namely, engineering design, management method, organizational system, building material, construction machine, technical, project personnel, operating environment, objective factor, project funding and schedule. For whole engineering, these factors form a factor group that lead to residential engineering quality problems.

In the engineering system, the group factors interact rather than independent individually, and make multi-effect to engineering qualities possibly, so, these factors can be named multi-effect factors. Obviously, the impact degree of multi-effect factors to engineering quality are different, if make the impact degree divided into five classes, larger(5), large(4), medium(3), small(2)and smaller(1), the12engineering experts give following corresponding values with every factors as table 2 .

Table. 2 The impact degree of multi-effect factors to residential engineering quality

\begin{tabular}{|c|c|c|c|c|c|c|c|c|c|c|}
\hline Design & $\begin{array}{c}\text { Managing } \\
\text { method }\end{array}$ & $\begin{array}{c}\text { Organization } \\
\text { system }\end{array}$ & Machine & Material & Technical & $\begin{array}{c}\text { Project } \\
\text { personnel }\end{array}$ & $\begin{array}{c}\text { Operating } \\
\text { environment }\end{array}$ & $\begin{array}{c}\text { Objective } \\
\text { factor }\end{array}$ & Funding & Schedule \\
\hline 4 & 3 & 3 & 3 & 3 & 4 & 4 & 4 & 4 & 2 & 2 \\
\hline 4 & 4 & 4 & 2 & 2 & 4 & 4 & 3 & 3 & 3 & 2 \\
\hline 5 & 3 & 3 & 1 & 4 & 4 & 5 & 3 & 5 & 4 & 3 \\
\hline 3 & 4 & 4 & 1 & 2 & 4 & 5 & 3 & 3 & 3 & 2 \\
\hline 3 & 4 & 3 & 3 & 2 & 4 & 5 & 3 & 3 & 2 & 2 \\
\hline 4 & 4 & 3 & 2 & 3 & 3 & 5 & 3 & 4 & 3 & 1 \\
\hline 3 & 4 & 3 & 3 & 3 & 4 & 4 & 3 & 2 & 2 & 3 \\
\hline 4 & 4 & 3 & 3 & 2 & 3 & 3 & 3 & 4 & 3 & 2 \\
\hline 5 & 4 & 4 & 3 & 3 & 4 & 4 & 3 & 5 & 2 & 3 \\
\hline 3 & 4 & 4 & 3 & 4 & 3 & 5 & 3 & 3 & 2 & 2 \\
\hline 2 & 4 & 3 & 2 & 2 & 4 & 3 & 3 & 2 & 3 & 1 \\
\hline 2 & 4 & 3 & 1 & 2 & 4 & 4 & 4 & 4 & 3 & 2 \\
\hline
\end{tabular}

\section{Analysis of Multi-effect Factors}

At present, the most common method to analyze the relationship of multi-effect factor is the adjacent matrix based on reduction to unity ${ }^{[3]}$. This method puts all data in to a matrix after unite them by a predefined rule ${ }^{[4]}$, and then obtain every factor's relative weight in factor group. According to every factor's relative weight, the impact degree of every multi-effect factor to management object can be determined. So, based above data in table 2 , the adjacent matrix of these 11 categories multi-effect factors can be established as following formula one based on reduction to unity.

$$
U=\left\{u_{i j}\right\}=\left|\begin{array}{lllllllllll}
0.8 & 0.6 & 0.6 & 0.6 & 0.6 & 0.8 & 0.8 & 0.8 & 0.8 & 0.4 & 0.4 \\
0.8 & 0.8 & 0.8 & 0.4 & 0.4 & 0.8 & 0.8 & 0.6 & 0.6 & 0.6 & 0.4 \\
1.0 & 0.6 & 0.6 & 0.2 & 0.8 & 0.8 & 1.0 & 0.6 & 1.0 & 0.8 & 0.6 \\
0.6 & 0.8 & 0.8 & 0.2 & 0.4 & 0.8 & 1.0 & 0.6 & 0.6 & 0.6 & 0.4 \\
0.6 & 0.8 & 0.6 & 0.6 & 0.4 & 0.8 & 1.0 & 0.6 & 0.6 & 0.4 & 0.4 \\
0.8 & 0.8 & 0.6 & 0.4 & 0.6 & 0.6 & 1.0 & 0.6 & 0.8 & 0.6 & 0.2 \\
0.6 & 0.8 & 0.6 & 0.6 & 0.6 & 0.8 & 0.8 & 0.6 & 0.4 & 0.4 & 0.6 \\
0.8 & 0.8 & 0.6 & 0.6 & 0.4 & 0.6 & 0.6 & 0.6 & 0.8 & 0.6 & 0.4 \\
1.0 & 0.8 & 0.8 & 0.6 & 0.6 & 0.8 & 0.8 & 0.6 & 1.0 & 0.4 & 0.6 \\
0.6 & 0.8 & 0.8 & 0.6 & 0.8 & 0.6 & 1.0 & 0.6 & 0.6 & 0.4 & 0.4 \\
0.4 & 0.8 & 0.6 & 0.4 & 0.4 & 0.8 & 0.6 & 0.6 & 0.4 & 0.6 & 0.2 \\
0.4 & 0.8 & 0.6 & 0.2 & 0.4 & 0.8 & 0.8 & 0.8 & 0.8 & 0.6 & 0.4
\end{array}\right|
$$

Here $u_{i j}$ is the normalized value, $i=12, \quad j=11$. If using factor analysis principle as a tool to deal with the matrix, and let every factor value as its weight $\omega_{j}$, then, the following result can be obtained.

$$
\omega=\left\{\omega_{i}, i=1-11\right\}=\{0.092,0.103,0.093,0.079,0.073,0.101,0.125,0.093,0.093,0.083,0.065\}
$$


Where, $\min \{\omega\}=\omega_{11}=0.065$.If selected it as the comparison base value, the multi-effect value $\delta_{j}$ of each factor respectively is as follow.

$\delta_{1}=1.42, \quad \delta_{2}=1.58, \quad \delta_{3}=1.43, \quad \delta_{4}=1.21, \quad \delta_{5}=1.12, \quad \delta_{6}=1.55, \quad \delta_{7}=1.92, \quad \delta_{8}=1.43, \quad \delta_{9}=1.43, \quad \delta_{10}=1.27, \quad \delta_{11}=1.00$

According to the relevant rule of factor analysis principle, the consistency test value should be less than 0.10 , and $\gamma=5.78$ if factors number $\mathrm{N}$ is $11^{[5]}$, so,

$$
\bar{\alpha}=\frac{1}{N} \sum \frac{\delta_{i}}{\omega_{i}}=15.4, \mathrm{R}=\frac{\bar{\alpha}-N}{N-1}=\frac{15.4-11}{11-1}=0.44, \beta=R / \gamma=\frac{0.44}{5.78}=0.0761 \angle 0.10
$$

It ca be seen from the analysis result that this analysis process meets the consistency test requirements of factors analysis principle ${ }^{[6]}$. On the bases of which, if rank all multi-effect factors according to the multi-effect value of each factor from small to large order, then, the below outcome can be obtained.

$$
\delta_{11}<\delta_{5}<\delta_{4}<\delta_{10}<\delta_{1}<\delta_{3}=\delta_{8}=\delta_{9}<\delta_{6}<\delta_{2}<\delta_{7}
$$

According to the order of all multi-effect factors, project managers can find the impact degree of every multi effect factor to residential engineering problems and take corresponding measure to solve engineering quality problems.

\section{Conclusion}

In order to understand residential engineering quality problems, finding their primary and secondary sources, and provide effective evidences for solving such problems, the paper analyzed the survey data about residential engineering quality problems, and found these problems' new features that often appear residential engineering in recent years. Based on statistics data of survey results, the relationship of multi-effect factors has also been analyzed and obtained following achievements.

(1) In recent years, the total residential engineering quality in China has a large improvement, but the residential quality level has a big difference in various regions.

(2) 24 kinds of major quality problems often appear in residential engineering, where, indoor air pollution is the most important quality problem.

(3)The factors that result in residential quality problems have 11 categories, where, the engineering schedule and inferior materials are the most multi-effect factors.

\section{Acknowledgement}

This study was supported by the national natural science foundation of China (Grant No. 71171081) and the natural science foundation of Beijing (Grant No. 9162014).

\section{References}

[1] Analysis on the residential engineering quality problems in China. http://ziliao.co188.com.

[2] Several suggestions about engineering quality and safety. http://www.mohurd.gov.cn/jsbfld.html.

[3] K.C. Gong, C.X. Wu: Adaptive Image Segmentation Algorithm Based on the Adjacency Matrix. Electronic Science and Technology Vol. 29 (2016), p. 66-75.

[4] H.Y.Zhong, J.T. Xiao: Apply Technology Acceptance Model with Big Data Analytics and Unity . International Conference on Software Engineering and Service Sciences (2015), p.19-24.

[5] D.F. Chen: Statistics Theory (Beijing Technology University Press Publications, Beijing 2017).

[6] L. Wang, X.Z. Xu.: Consistent Variable Selection Via The Optimal Discovery Procedure in Multiple Testing. Communications in Statistics - Theory and Methods, Vol. 46 (2017), p. 6303-6322. 